\title{
DETERMINACIÓN EXPERIMENTAL DE LA FORMABILIDAD DE LÁMINAS DE ACERO SG295 MEDIANTE SUS PROPIEDADES TENSILES
}

\section{EXPERIMENTAL DETERMINATION OF THE FORMABILITY OF SG295 STEEL BLADES THROUGH THEIR TENSILE PROPERTIES}

\author{
MSc. Claudia Liliana Casadiego Peralta", MSc. Jhon Erickson Barbosa Jaimes" \\ PhD. Ismael Humberto García Páez ${ }^{*}$ \\ * Universidad Francisco de Paula Santander, Grupo de Investigación GIDIMA. \\ Av Gran Colombia 12E-96 B Colsag, Cúcuta, Norte de Santander, Colombia. \\ $037-5776655$ Ext. 162 \\ E-mail: $\{$ lilianacasadiego, jhonericksonbj, ismaelhumbertogp $\} @$ ufps.edu.co
}

Resumen: En la presente investigación experimental se determinaron los límites de formabilidad de láminas de acero SG295 de $2.2 \mathrm{~mm}$ de espesor, las cuales son utilizadas en la manufactura de cilindros para gas. Se realizó inicialmente una caracterización química y un análisis metalográfico. Las propiedades mecánicas determinadas fueron la resistencia a la tracción, el límite elástico mediante la aplicación de ensayos de tracción (ASTM E 8M) y la ductilidad, la cual se determinó mediante el alargamiento hasta fractura. El índice de resistencia al angostamiento del material se midió con el ensayo ASTM E 517 que permite determinar el coeficiente de anisotropía, r. La capacidad del material para distribuir la deformación uniformemente se cuantificó mediante el coeficiente de endurecimiento por deformación, n, según la norma ASTM E 646. Los resultados muestran una ductilidad aceptable para un acero al carbono y un comportamiento anisotrópico adecuado para operaciones de embutido.

Palabras clave: Formabilidad, tracción, anisotropía, embutido, acero.

\begin{abstract}
In the present experimental research, the formability limits of SG295 steel sheets with $2.2 \mathrm{~mm}$ of thick were determined. This material is used in the manufacture of gas cylinders. Chemical characterization and metallographic analysis was initially performed. The mechanical properties determined were the tensile strength, the yield strength by applying tensile testing (ASTM E 8M) and ductility, which was determined by the elongation to fracture. The narrowing resistance index was measured with test ASTM E 517, which determines the anisotropy coefficient, $r$. The ability of material to distribute evenly deformation was quantified by the strain hardening coefficient, n, according to ASTM E 646 standard. The results show an acceptable ductility for a carbon steel and a suitable anisotropic behavior for operations of deep drawing.
\end{abstract}

Keywords: Formability, strength, anisotropy, deep drawing, steel. 


\section{INTRODUCCIÓN}

La formabilidad es la medida de la deformación que puede presentarse en una lámina metálica antes de fracturarse o sufrir encuellamiento. Esta propiedad no puede definirse en función de un solo parámetro, sino que depende entre otros, del proceso de conformado en particular (por ejemplo el embutido y el estirado), del espesor de la chapa, y en gran medida de las propiedades intrínsecas del material. Estas propiedades influyen directa o indirectamente en la formabilidad y en la calidad del producto, y son: la resistencia última, el límite elástico, la ductilidad, el coeficiente de anisotropía, $\mathrm{r}$ y el exponente de endurecimiento por deformación, n. Este último está relacionado con la capacidad de la chapa para distribuir la deformación uniformemente (operaciones de estirado) (Gedney, 2013a). De ahí que esta propiedad sea de gran interés técnico y económico para las empresas manufactureras, toda vez que permite predecir el desempeño de la chapa metálica en este tipo de operaciones.

Los ensayos que miden la conformabilidad se dividen en dos tipos básicos: intrínsecos y simulativos. Los ensayos intrínsecos miden las propiedades básicas del material que pueden relacionarse con su conformabilidad. Por otro lado, los ensayos simulativos someten el material a deformación que se asemeja estrechamente a la deformación que ocurre en una operación de conformación real (Taylor, 1988, Torres et al., 2013).

Los ensayos intrínsecos proporcionan información global que es insensible al espesor y condición superficial del material. El más importante y ampliamente usado es el ensayo de tracción uniaxial ASTM E8, el cual provee valores de muchas propiedades del material para un amplio rango de operaciones de conformación (Gedney, 2013a).

Los ensayos simulativos proporcionan información específica y limitada que es normalmente sensible al espesor, a la condición superficial, a la lubricación, a la geometría y al tipo de herramienta. Esta información se relaciona a sólo un tipo de operación de conformación (Roger, 2005; Rodríguez O et al., 2012).

Entonces, el propósito fundamental de este trabajo ha sido medir experimentalmente la formabilidad de láminas de Acero SG295 de $2.2 \mathrm{~mm}$ de espesor, utilizadas por la empresa en la manufactura de cilindros para gas. Para tal fin, diversas probetas fueron sometidas a ensayos estandarizados de tracción ASTM E 8M, ASTM E 517 y ASTM E646.

Del ensayo de tracción se obtuvieron propiedades importantes como: la resistencia a la tracción, $\sigma \mathrm{u}$, el límite elástico convencional, $\sigma y \quad(0.2 \%)$, el alargamiento hasta fractura, A50, y el exponente de endurecimiento por deformación, n. Finalmente, se determinó el coeficiente de anisotropía normal, $r$.

\section{MATERIALES Y MÉTODOS}

\subsection{Composición química y estudio metalográfico}

La determinación cuantitativa de los elementos aleantes presentes en la composición de las láminas se determinó usando un espectrómetro de emisión óptica (OES, Optical emisión Spectrometer) marca BRUKER, modelo Q8 MAGELLAN.

Para la preparación de las muestras metalográficas, se procedió en conformidad con la norma ASTM E 3 que lleva por título "Standard Practice for Preparation of Metallographic Specimens". El revelado de la microestructura se hizo mediante ataque con nital al $2 \%$ y la observación se realizó en un microscopio óptico de luz reflejada marca ZEISS, modelo Axio Scope.A1.

\subsection{Ensayo de tracción}

Las muestras para la fabricación de las probetas fueron obtenidas de la empresa CINSA. Se estableció como criterio para la preparación de las probetas, lo estipulado en la norma ASTM E $8 \mathrm{M}$ que lleva por título "Standard Test Methods for Tension Testing of Materials", como se describe a continuación: Las probetas para este ensayo fueron fabricadas mediante troquelado, con una matriz con las medidas definidas en la norma descrita anteriormente (Figura 1). Se fabricaron probetas con orientaciones con respecto de la última laminación de $0^{\circ}, 45^{\circ}$ y $90^{\circ}$.

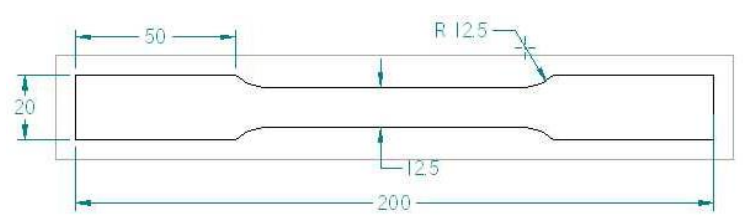

Fig. 1. Geometría y dimensiones de la probeta plana para tracción, tipo A. (ASTM E 8M), unidades en milímetros. (Barbosa, 2008) 
Las propiedades a tracción fueron calculadas a partir de los datos obtenidos por la máquina universal de ensayos de tracción/compresión marca SHIMADZU, modelo AG 250.

Se calcularon las siguientes propiedades: Resistencia a la tracción, $\sigma_{\mathrm{u}}$, Límite elástico convencional, $\sigma_{\mathrm{y}(0.2 \%)}$, y Alargamiento, $\mathrm{A}_{(50)}$.

\subsection{Exponente de endurecimiento por deformación, $n$}

Para determinar el exponente de endurecimiento por deformación, $n$, se siguieron las especificaciones dadas por la norma ASTM E 646 que lleva por título "Standard Test Method for Tensile Strain-Hardening Exponents (n-Values) of Metallic Sheet Materials". Dicho exponente $n$ fue determinado a partir de una representación matemática empírica sobre el rango de interés de la curva esfuerzo real vs deformación real, obtenida del ensayo de tracción uniaxial.

La representación matemática empleada en este método es aplicable a metales para los cuales el comportamiento plástico obedece a una curva potencial de la forma:

$\sigma_{r}=\mathrm{k}^{*} \in^{\mathrm{n}}$

Donde:

$$
\begin{aligned}
& \sigma_{r}=\text { Esfuerzo real. } \\
& \epsilon=\text { Deformación real. } \\
& k=\text { Coeficiente de resistencia. } \\
& n=\text { Coeficiente de } \\
& \text { endurecimiento por deformación. }
\end{aligned}
$$

El procedimiento para determinar $\boldsymbol{n}$ se indica a continuación:

A partir de datos del ensayo de tracción, se determinó el esfuerzo real $\left(\sigma_{\mathrm{r}}\right)$ y la deformación real $(\epsilon)$. El esfuerzo real se calcula sobre la base del área transversal instantánea, A. Es decir:

$\sigma_{r}=\mathrm{F} / \mathrm{A}$

Si el encuellamiento no ha tenido lugar, resulta:

$\sigma_{r}=\sigma^{*}(1+\not \subset)$

Donde, $\sigma$ es el esfuerzo de ingeniería y $\not \subset$ es la deformación de ingeniería. La deformación real corresponde al logaritmo natural de la relación entre la longitud de calibración instantánea, L, a la longitud de calibración inicial, $\mathrm{L}_{0}$. Esto es,

$$
\begin{aligned}
& \in=\ln \left(\mathrm{L} / \mathrm{L}_{\mathrm{O}}\right), \text { ó } \\
& \epsilon=\ln (1+\not \subset)
\end{aligned}
$$

A continuación, se seleccionan los pares esfuerzo real-deformación real $\left(\sigma_{\mathrm{r}}, \in\right)$ del intervalo de interés, que corresponde a la región plástica, es decir, entre el límite elástico y el esfuerzo máximo (inicio del encuellamiento).

Los pares elegidos son representados en un diagrama $\log \sigma_{\mathrm{r}}$ vs $\log \in$. Esto corresponde a la linealización de la curva de potencia $\sigma_{\mathrm{r}}=\mathrm{k}^{*} \in \mathrm{n}$, al tomar logaritmo de esta ecuación resulta:

$\log \sigma_{\mathrm{r}}=\log \mathrm{k}+\mathrm{n} \log \in$

Siendo la ecuación anterior una recta de la forma

$\mathrm{y}=\mathrm{a}+\mathrm{bx}$

$=\mathrm{n}$ y $\mathrm{K}=$ antilogaritmo de a. La ecuación del esfuerzo real será obtenida directamente por ajuste a la curva representada en una hoja de cálculo, Figura 2.

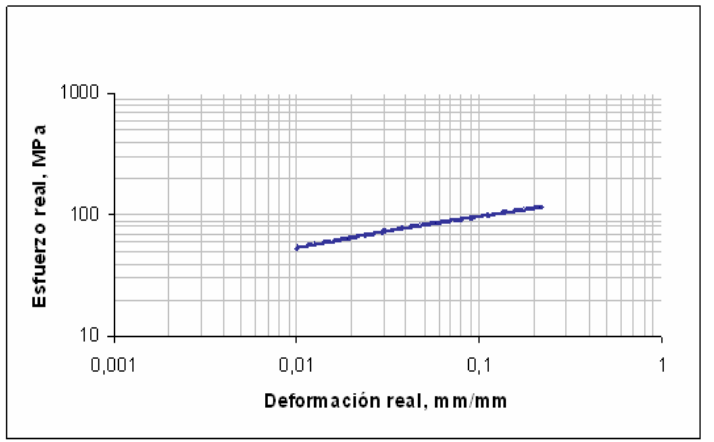

Fig. 2. Curva Esfuerzo real-Deformación real (log-log). (Barbosa, 2008)

\subsection{Coeficiente de anisotropía, $r$}

Para su determinación se siguieron las especificaciones dadas por la norma ASTM E 517. Que lleva por título "Standard Test Methods for Plastic Strain Ratio r for Sheet Metal”.

Se fabricaron 4 probetas por cada dirección de laminación para ser ensayadas hasta una deformación del 15\%. Con una velocidad del ensayo en $4 \mathrm{~mm} / \mathrm{min}$. 
Se midieron las deformaciones en probetas orientadas a $0^{\circ}, 45^{\circ} \mathrm{Y} 90^{\circ}$ respecto de la dirección de laminación y se determinó la anisotropía normal así:

$r=\frac{\epsilon_{w}}{\epsilon_{t}}=\frac{\ln \left(w_{0} / w_{f}\right)}{\ln \left(l_{f} w_{f} / l_{0} w_{0}\right)}$

$R_{\text {prom }}=\frac{r 0+2 r 45+r 90}{4}$

Donde:

$\mathrm{R}_{\text {prom }}$ es el valor de la anisotropía normal.

$w_{o} y w_{f}$ corresponden a las medidas del ancho de la probeta al inicio $y$ al final del ensayo respectivamente.

$l_{o} y l_{f}$ corresponden a las medidas del largo de la longitud marcada en la probeta al inicio y al final del ensayo respectivamente. (Figura 3).

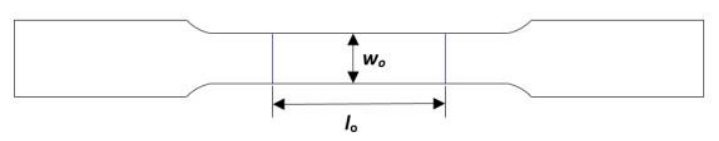

Fig. 3. Longitudes de calibración para medidas iniciales en el ensayo ASTM E517.

\section{RESULTADOS Y DISCUSIÓN}

\subsection{Composición química}

La caracterización química de la muestra de acero SG295 se presenta en la Tabla 1.

Los valores para los componentes principales como el carbono, manganeso, silicio, fósforo y azufre, están entre los límites establecidos para las láminas de acero SG 295 de acuerdo a la norma JIS 3116 referenciada por los fabricantes (BEBON, 2016; ACESCO, 2012).

De acuerdo a los porcentajes en peso de componentes principales registrados en la tabla 1 , se pueden considerar como acero al carbono y más específicamente como acero dulce (Askeland et al., 2013).

Tabla 1: Resultados del análisis químico del acero $\underline{S G 295}$

\begin{tabular}{|c|c|}
\hline $\begin{array}{l}\text { Elemento de } \\
\text { aleación }\end{array}$ & $\begin{array}{l}\text { SG295 } \\
\text { (\% peso) }\end{array}$ \\
\hline Carbono (C) & 0.168 \\
\hline Silicio $(\mathrm{Si})$ & 0.0032 \\
\hline Manganeso (Mn) & 0.694 \\
\hline Fósforo (P) & 0.017 \\
\hline Azufre (S) & 0.0048 \\
\hline Cromo (Cr) & 0.021 \\
\hline Molibdeno (Mo) & 0.0068 \\
\hline Níquel (Ni) & 0.021 \\
\hline Cobre $(\mathrm{Cu})$ & 0.012 \\
\hline Aluminio $(\mathrm{Al})$ & 0.033 \\
\hline Arsénico (As) & 0.0024 \\
\hline Cobalto $(\mathrm{Co})$ & 0.0029 \\
\hline Niobio $(\mathrm{Nb})$ & 0.0014 \\
\hline Plomo $(\mathrm{Pb})$ & 0.0028 \\
\hline Antimonio (Sb) & 0.0011 \\
\hline Estaño (Sn) & 0.0011 \\
\hline Vanadio (V) & 0.0026 \\
\hline
\end{tabular}

\subsection{Metalografía}

La estructura metalográfica del acero estudiado se muestra en la Figura 4. En ella se observa que la microestructura del acero SG295 es ferrita $(90 \%)$ y perlita $(10 \%)$ de grano equiaxial muy fino (tamaño de grano 10), no se observa alargamiento del grano en la dirección de la laminación, típico de un producto del laminado en caliente con posterior recristalización. Se observa gran cantidad de pequeños glóbulos de carburo retenido.

El tamaño de grano es inversamente proporcional a las medidas de resistencia y ductilidad en las hojas metálicas como lo expresa Kalpakjian y Schmi, 2008. Así, pues, el pequeño tamaño de grano de esta muestra supone buen comportamiento de estas propiedades.

\subsection{Propiedades mecánicas}

En la Tabla 2 se indican los resultados del límite elástico convencional, $\boldsymbol{\sigma}_{\mathbf{y}(\mathbf{0 . 2} \%)}$ y la resistencia a la tracción para cada uno de los espesores de lámina estudiados según diversas orientaciones con relación a la dirección de la última laminación.

Como se observa en la Tabla 2, las propiedades dependen de la orientación en que se midieron. Esto es de esperar debido a la característica inherente de anisotropía de los materiales de ingeniería. 


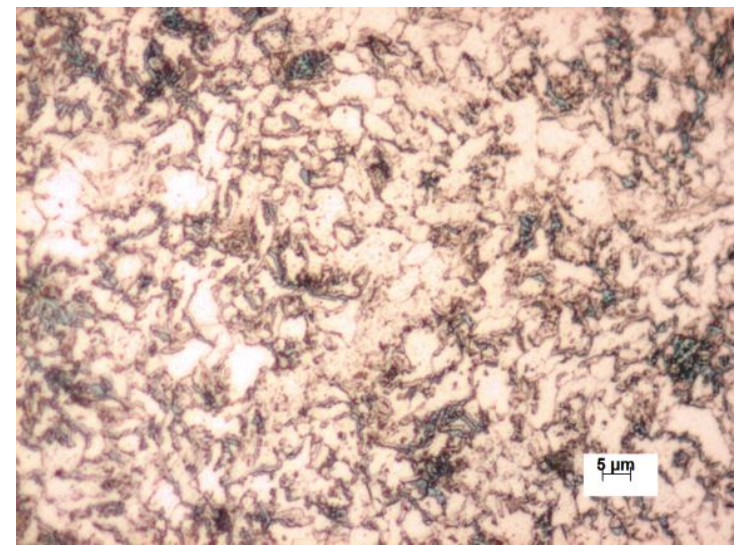

Fig. 4. Micrografía de una muestra de acero SG295. Magnificación 1000X.

Tabla 2: Resultados del límite elástico y resistencia a la tracción del acero SG295

\begin{tabular}{|c|c|c|}
\hline & $\begin{array}{c}\text { Límite elástico } \\
\text { convencional } \\
(\mathbf{M P a})\end{array}$ & $\begin{array}{c}\text { Resistencia a la } \\
\text { tracción } \\
\text { (MPa) }\end{array}$ \\
\hline $0^{\circ}$ & 357.5 & 431.5 \\
\hline $45^{\circ}$ & 343 & 456 \\
\hline $90^{\circ}$ & 346.1 & 462.25 \\
\hline & $\mathbf{3 4 8 . 9 \pm 7 . 6}$ & $\mathbf{4 4 9 . 9 \pm 1 6 . 3}$ \\
\hline
\end{tabular}

El límite elástico convencional del $0.2 \%, \sigma_{\mathrm{y}},(\mathbf{0 . 2} \%)$, presenta un valor promedio de $348.9 \mathrm{MPa}$, el cual es muy superior a los 295MPa establecido para las láminas de acero SG 295 de acuerdo a la norma JIS 3116 referenciada por los fabricantes (BEBON, 2016; ACESCO, 2012).

El valor promedio de la resistencia a la tracción es de 449.9 MPa. Este valor es ligeramente superior a los $440 \mathrm{MPa}$ establecidos para las láminas de acero SG 295 de acuerdo a la norma JIS 3116 referenciada por los fabricantes (BEBON, 2016; ACESCO, 2012).

El alargamiento hasta fractura es una medida de la ductilidad del material, y por tanto está relacionado con la capacidad de conformado de la lámina metálica (Askeland, 2013; Newel, 2009). En la Tabla 3 se indican los valores del porcentaje de alargamiento hasta fractura con respecto a la longitud calibrada de $50 \mathrm{~mm}$; valores que fueron obtenidos en el ensayo a tracción según diversas orientaciones.

El valor promedio del alargamiento hasta fractura es de $24.89 \%$, ligeramente por debajo del $26 \%$ establecido para la lámina de acero SG 295 de acuerdo a la norma JIS 3116 referenciada por los fabricantes (BEBON, 2016; ACESCO, 2012).

El exponente de endurecimiento por deformación es una medida de la rapidez con que un metal se vuelve más resistente y fuerte (Gedney, 2013a). Así, en un material con un valor alto de n, el espesor se reduce más uniformemente en operaciones de estirado y la elongación es más grande antes de aparecer encuellamiento, y por tanto es un indicativo de buena formabilidad (Schey, 2002; Kalpakjian y Schmid, 2008; Askeland, 2013, Caballero A. et al., 2013).

\section{Tabla 3: Resultados del alargamiento hasta fractura del acero SG295}

\begin{tabular}{|c|c|}
\hline Orientación & $\begin{array}{c}\text { Alargamiento hasta } \\
\text { fractura, } \boldsymbol{A}_{\mathbf{5 0}} \\
(\boldsymbol{\%})\end{array}$ \\
\hline $0^{\circ}$ & 24.56 \\
\hline $45^{\circ}$ & 28.84 \\
\hline $90^{\circ}$ & 21.26 \\
\hline & $\mathbf{2 4 . 8 9} \pm \mathbf{3 . 8 0}$ \\
\hline
\end{tabular}

Los resultados obtenidos de $\boldsymbol{n}$, se indican en la Tabla 4, y están discriminados según la orientación respecto a la última dirección de laminación.

Los valores de $\boldsymbol{n}$ en sus tres orientaciones son muy similares y presentan un promedio de 0.233. Aunque para la orientación de $45^{\circ}$ se tiene el valor más bajo, éste coincide con el 0,22 referido por Gedney, 2013a para el acero al $0.2 \%$ de carbono laminado en caliente, y está ligeramente por debajo de los 0,26 reportados por Kalpakjian y Schmid, 2008 para el acero al bajo carbono recocido.

Tabla 4. Resultados del exponente de endurecimiento por deformación para el acero

\begin{tabular}{|c|c|}
\hline Orientación & $\begin{array}{c}\text { Exponente de } \\
\text { endurecimiento por } \\
\text { deformación, } \boldsymbol{n}\end{array}$ \\
\hline $0^{\circ}$ & 0.23 \\
\hline $45^{\circ}$ & 0.225 \\
\hline $90^{\circ}$ & 0.245 \\
\hline & $\mathbf{0 . 2 3 3 \pm 0 . 0 1 0}$ \\
\hline
\end{tabular}

La anisotropía normal define el comportamiento de la chapa o lámina metálica en cuanto al adelgazamiento en operaciones de estirado y embutido profundo. Ésta se adquiere en el proceso de formación de la lámina y está definida por la orientación preferencial de los granos y por la 
alineación de impurezas e inclusiones a través del espesor (Kalpakjian y Schmid, 2008). Además, indica la capacidad de la hoja de metal para resistir el adelgazamiento o engrosamiento cuando se somete a operaciones de embutido (Gedney, 2013a, 2013b).

Los resultados obtenidos de $\boldsymbol{r}$ se indican en la Tabla 5 y están discriminados según la orientación respecto a la última dirección de laminación y su respectivo valor de $\mathrm{R}_{\text {prom. }}$

\section{Tabla 5. Resultados del coeficiente de anisotropía para el acero $S G 295$}

\begin{tabular}{|c|c|}
\hline Orientación & $\begin{array}{c}\text { Coeficiente de } \\
\text { anisotropía }, \boldsymbol{r}_{(\mathbf{1 5})}\end{array}$ \\
\hline $0^{\circ}$ & 0.64 \\
\hline $45^{\circ}$ & 1.41 \\
\hline $90^{\circ}$ & 3.12 \\
\hline $\mathbf{R}_{\text {prom }}$ & $\mathbf{1 . 6 5}$ \\
\hline
\end{tabular}

En la Tabla 5 puede verse que los valores en sus tres orientaciones difieren completamente, y que la orientación de $90^{\circ}$ presenta el valor más alto. El valor del $\mathrm{R}_{\text {prom }}$ está ligeramente por encima de los rangos reportados por Kalpakjian y Schmid, 2008, de 0.9-1.2 para los aceros de baja aleación y de 0.81.0 para el acero laminado en caliente. A su vez coinciden con el rango de 1.0-1.6 referido por Gedney, 2013a, para el acero al $0.2 \%$ de carbono laminado en caliente.

Los resultados muestran que aunque en el análisis metalográfico no se observa denota un alargamiento preferencial de los granos, si se presenta un comportamiento anisotrópico. Sin embargo, esta anisotropía no se refleja ampliamente en las otras propiedades mecánicas determinadas.

En la figura 5 se grafican los valores del coeficiente de anisotropía en función del ángulo respecto de la dirección de laminación para el acero SG295. Se observa un incremento a medida que se aumenta el ángulo, con valores promedio y de desviación estándar de $0.64 \pm 0.16 ; 1.41 \pm 0.19$ y $3.12 \pm 0.68$ respectivamente para los ángulos de $0^{\circ}, 45^{\circ}$ y $90^{\circ}$. También hay una marcada dispersión de los datos, lo que es asociado por algunos autores a la textura cristalográfica del material (Serenelli et al., 2008; Newel, 2009).

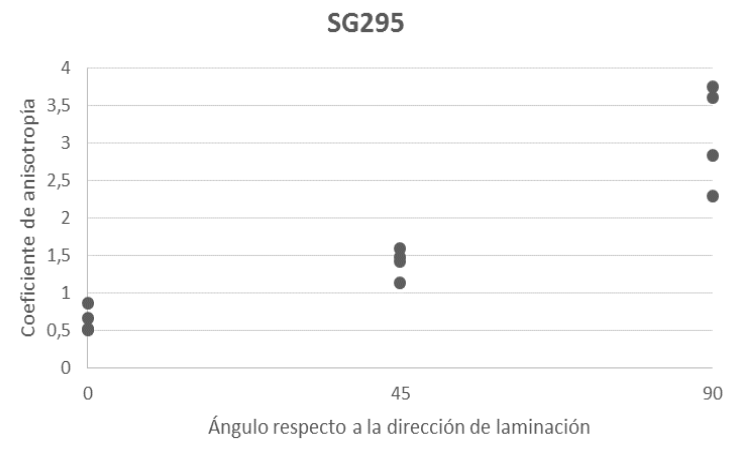

Fig. 5. Valores del coeficiente de anisotropía en función del ángulo respecto de la dirección de laminación para el acero SG295.

\section{CONCLUSIONES}

La formabilidad de la lámina de acero SG295 fue determinada experimentalmente y fue relacionada con el análisis de la composición química, el análisis metalográfico y las propiedades mecánicas.

El acero SG295 analizado es un acero al carbono con ferrita (90\%) y perlita $(10 \%)$ de grano equiaxial muy fino (tamaño de grano 10).

Las propiedades mecánicas derivadas del ensayo de tracción presentan una diferencia de $101 \mathrm{MPa}$ entre los valores promedio del límite elástico convencional y la resistencia a la fractura. Este comportamiento está relacionado con una moderada ductilidad del material, lo que se confirma con el valor del alargamiento hasta fractura promedio de $24.89 \%$ en $50 \mathrm{~mm}$.

El valor del exponente de endurecimiento por deformación, $\boldsymbol{n}$, no muestra una tendencia clara, ya que los valores obtenidos fueron muy similares para cada orientación, pero su valor promedio (0.233) concuerda con el de los aceros al carbono laminados en caliente.

Los valores del coeficiente de anisotropía $r$ presentan, una alta dispersión en los datos, especialmente para la orientación de $90^{\circ}$.

\section{RECONOCIMIENTO}

Los autores expresan su agradecimiento a la Universidad Francisco de Paula Santander por la financiación del proyecto a través del Fondo de Investigaciones Universitarias FINU.

\section{REFERENCIAS}


ACESCO (2012). Catálogo de productos ACESCO. Recuperado de https://issuu.com/jordan021294/docs/catalogo deproductos 2012

Askeland, F. y Wright, W. (2013). Ciencia e ingeniería de los materiales, $6^{\circ}$ edición. Thomson

ASTM E 3-01. Standard Pratice for Preparation of Metallographic Specimens, 2001

ASTM E 517- 10. Standard Test Methods for Plastic Strain Ratio $r$ for Sheet Metal, 2010

ASTM E 646 - 00. Standard Test Methods for Tensile Strain-Hardening Exponents (n Values) of Metallic Sheet Materials, 2000

ASTM E 8M- 13a. Standard Test Methods for Tension Testing of Metallic Materials [Metric], 2013

Barbosa, J. (2008). Estudio Experimental de la formabilidad de chapas de aluminio fabricadas por la empresa Aluminios ONAVA. Tesis de Maestría, Universidad Nacional Experimental del Táchira. San Cristóbal, Venezuela.

BEBON (2016). Catálogo de productos BEBON. Recuperado de http://www.steel-platesheet.com/Steel-plate/JIS/SG295.html

Caballero Amaury, Velasco Gabriel, Pardo García A. (2013). DIFFERENTIATIONS OF OBJECTS IN DIFFUSE DATABASES. Revista colombiana de tecnologías de Avanzada. 2 (22). Pág. 131 - 137.

Gedney R. (2013). Measuring the Plastic Strain Ratio of Sheet Metals. ADMET, Inc., Norwood, MA 02062 USA

Gedney R. (2013). Tensile Testing for Determining the Formability of Sheet Metals. ADMET, Inc., Norwood, MA 02062 USA

Kalpakjian, S. y Schmid, S. (2008). Manufactura, Ingeniería y Tecnología, $5^{a}$ edición. Prentice Hall

Metals Handbook (1973). Metallography, Structures and Phase Diagrams, Vol 7, 8th Edition. ASM.

Metals Handbook (1988). Forming and Forming, vol. 14, 9th Edition. ASM.

Metals Handbook (1988). Forming of Aluminum Alloy Sheet, Vol 14, 9th Edition. ASM.

Newel, J. (2009). Ciencia de materiales, aplicaciones en ingeniería, $1^{\circ}$ edición. Alfaomega
Roger, A. (2005). Deformation Characteristics of Stainless Steels. Tesis doctoral. Lulea University of Technology, Lulea. Sweden.

Rodríguez Oscar Oswaldo, Pineda Pinto Ronald Fernando, Cárdenas Pedro Fabián. (2012). Herramientas EJS 3D/MATLAB para el control del sistema no lineal aplicado al péndulo invertido sobre carro deslizante. Revista colombiana de tecnologías de Avanzada. 1 (19). Pág. 28 - 34.

Panich, S., Barlat, F, Uthaisangsuk, V. Suranuntchai, S. y Jirathearanat, S. (2013) Experimental and theoretical formability analysis using strain and stress based forming limit diagram for advanced high strength steels. Materials and Design. 51, 756-766

Schey, A. (2002). Procesos de Manufactura, $3^{\text {a }}$ edición. McGraw-Hill

Serenelli, M., Bertinetti, M. y Signorelli, J. (2008). Influencia de la textura cristalográfica en la dispersión de coeficientes de lankford en una chapa de acero galvanizada de bajo carbono. Mecánica Computacional Vol XXVII, 9931001.

Spence, M. Chan, A. y Capson, D. (2005). Automotive Sheet Metal and Grid Digitizing Solutions. Computer-Aided Design \& Applications, Vol. 2, Nos. 1-4, 2005.

Taylor, B. (1988). Formability Testing of Sheet Metals. Metals Handbook, vol. 14, 9th Edition. ASM.

Torres Clayton José, Archila John Faber, Tronco Mário Luiz, Becker Marcelo, Viera Porto Arthur José, Tiberti Alexander José. (2013). Estudio cinemático de una plataforma robótica para agricultura. Revista colombiana de tecnologías de Avanzada. 2 (22). Pág. 131 $-137$. 